\title{
USE OF THE PASS-THROUGH METHOD TO SOLVE SOUND RADIATION PROBLEMS OF A SPHERICAL ELECTRO-ELASTIC SOURCE OF ZERO ORDER
}

\author{
Oleksii Korzhyk \\ Department of Acoustic and Multimedia Electronic Systems ${ }^{l}$ \\ Sergey Naida \\ Department of Acoustic and Multimedia Electronic Systems ${ }^{I}$ \\ Sergii Kurdiuk \\ Department of Marine Corps Institute \\ National University «Odessa Maritime Academy» \\ 8 Didrikhson str., Odessa, Ukraine, 65029 \\ Valeriia Nizhynska $\bowtie$ \\ Department of Acoustic and Multimedia Electronic Systems ${ }^{l}$ \\ nvv0807190-ames21@lll.kpi.ua \\ Maxim Korzhyk \\ Department of Acoustic and Multimedia Electronic Systems ${ }^{l}$ \\ Anton Naida \\ Department of Acoustic and Multimedia Electronic Systems ${ }^{l}$ \\ ${ }^{I}$ National Technical University of Ukraine «Igor Sikorsky Kyiv Polytechnic Institute» \\ 37 Peremohy ave., Kyiv, Ukraine, 03056
}

$\triangle$ Corresponding author

\begin{abstract}
In the article was solved the problem of radiation of a sound by the electroacoustic transducer which is executed in the form of a thin spherical cover, using a pass-through method. The outer and inner surfaces of the shell are completely electroded.

The application of this method provides an opportunity to avoid inaccuracies that arise during the traditional formulation of boundary conditions for acoustic mechanical fields, the use of equivalent substitution schemes and the absence of boundary conditions for the electric field in general. Given methodology eliminates these shortcomings by applying conjugation conditions, taking into account the types of electroding of the surfaces of piezoceramic transducers, the introduction of boundary conditions for current and voltage. The results of the solution demonstrate the high capabilities of this pass-through method, in terms of taking into account the peculiarities of determining the characteristics of these fields, values and dependences of the main complex characteristics of the electroelastic transducer, and auxiliary material constants of the piezoelectric material.

The proposed approach is relevant, because it allows to increase the reliability of modeling the operating conditions of acoustic transducers in the context of wave problems of acoustics. Aim is to enhance the range of performances and build algorithms solving problems of stationary mode hydroelectroelasticity sound radiation. The expected results are presented in terms of improving approaches to studying the features of the oscillatory process of the active elements of sound-emitting systems and the accompanying effects of the transformation of interconnected fields involved in the formation of the acoustic signal in the liquid.
\end{abstract}

Keywords: acoustics, field interaction, connectivity electroelastic radiation mode, electroacoustic transducer, hydroelectric.

DOI: $10.21303 / 2461-4262.2021 .001292$

\section{Introduction}

Pass-through formulations in the problems of mechanics and acoustics were initiated as a separate class of piezoelectric problems based on the provisions of Maison's physical acoustics [1] and the scientific schools of NASU academicians [2]. The important achievements of the founders include, first of all, the monograph [2], which substantiates the use of such productions in theoretical 
and applied issues of electroacoustic transducers of sound and ultrasound ranges. The convenience of such formulations in relation to the possibilities provided by them for in-depth study of the spatial and energy characteristics of electroacoustic piezoceramic transducers even leads to the popularization of the initiated approaches of the end-to-end problem (for example, source materials [3]).

Further development of theoretical and practical aspects of construction and use of electroelastic systems operating in conditions of static and dynamic deformation, was aimed at the problems of wave acoustics, theory and practice of development of sonar transducers and antennas. In this case, as examples of works on this topic include articles [4-10]. The work of followers, in accordance with the tasks of applied acoustics, expanded the physical aspects of pass-through stationary and non-stationary productions, supplementing them with Kirchhoff-Lev hypotheses, enriched the models with technological elements such as screens [10], elastic sealing layers, [11] orientations, liquid and solid fillers [12] and others. Special mention should be made of the work [2], which initiates the process of solving, systematizing and ordering the electroelastic models of transducers of certain canonical forms.

An attractive feature of the pass-through approach methodology is to take into account and visually predict the spatial properties of transducers by using individual modes, or combinations thereof. This is due to the peculiarities of the stress-strain state of the transducers design, types of electroding, kinematic and force conditions of conjugation.

It so happened that until recently more attention was paid to cylindrical multimode transducers and their systems, [9-11, 13]. Spherical, rod and bending transducers are somewhat less covered. At the same time, the frequency characteristics of the eigenforms of cylindrical and spherical shells, the features of electroding and electrical boundary conditions are of special interest. These issues are partially considered in [2, 4, 6, 14-16].

Expanding the range of pass-through statements [15-20] should lead to a more correct consideration of the operating conditions of electroelastic transducers, but requires a more thorough understanding of the interaction of acoustic, mechanical and electrical factors. Therefore, due to this, the physical aspects of end-to-end productions can be successfully used in the creation of models of receiving and radiating sonar systems.

All the above areas and work require further development, improvement and systematization.

Thus, the proposed «pass-through» approach is relevant and modern, because it allows to increase the reliability of modeling the operating conditions of acoustic transducers in the context of wave problems of acoustics with the replacement of boundary conditions by acoustic-mechanical fields - conjugation conditions and electrical boundary conditions. Aim is to enhance the range of performances and build algorithms solving problems of stationary mode hydroelectroelasticity sound radiation. The expected results are presented in terms of improving approaches to studying the features of the oscillatory process of the active elements of sound-emitting systems and the accompanying effects of the transformation of interconnected fields involved in the formation of the acoustic signal in the liquid.

\section{Materials and methods}

The problem under consideration is based on theoretical materials of the basic provisions of electric elasticity $[2,6]$ in terms of involvement in the solution: equations of motion of points on the surface of a thin sphere (Newton's second law), equations of state for piezoceramics, generalized Hooke's law, Cauchy relations and the Helmholtz equation for the acoustic field.

The solution is performed using generalized boundary conditions for acoustic, mechanical and electric fields using traditional methods of mathematical physics (Fourier method), vector analysis and orthogonal properties of spherical functions.

The work is structured in accordance with the order of solving traditional problems of sound scattering, reception and radiation $[10-12,15]$.

\section{1. The formulation of problem}

Suppose that a spherical electroacoustic transducer-emitter, supplied by a closed thin electrostress shell with radial polarization, is placed in the working medium, which is represented 
by an ideal liquid (Fig. 1). A spherical $O, r, \varphi, \theta$ and rectangular $O, X, Y, Z$ coordinate system is introduced into the medium, the centers of which coincide with the phase and geometric centers of the sphere (point $O$ ); the radius of the inner surface of the shell $-R$, external $-R_{1}$; shell wall thickness $-h_{s}=R_{1}-R$. In the volume of the shell is a vacuum. From the outside - a liquid with the density $\rho_{0}$ and speed of sound $c_{0}$. The inner $(r=R)$ and outer $\left(r=R+h_{s}\right)$ surfaces of the shell are completely electroded.

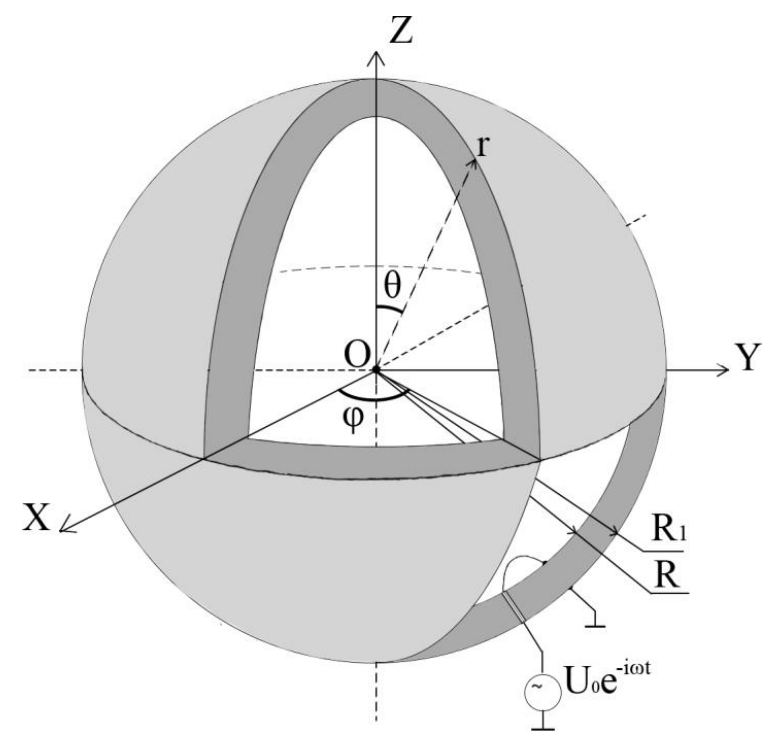

Fig. 1. Design scheme of a hollow sphere polarized by thickness

In the mode of radiation of the monochromatic signal, when performing the inequality $h_{s} \ll R$, the specified conditions of the transducer and the type of surface electroding [2, 14] allow to assume that:

- mechanical tensions $\sigma_{i j}$ and deformations $\varepsilon_{i j}$ (where $i, j$ single orts) - constant in the thickness of the shell wall (i.e. in the coordinate $r$ );

- the radial component of the electric field vector $E_{r}$ in the direction of polarization of the shell in the gap $h_{s}$ is also constant;

- disturbance and formation of mechano-acoustic fields - corresponds to the zero mode of oscillations of the sphere.

The inner and outer surfaces of the spherical transducer are connected [6] to a fixed frequency generator and together with it and the elements of the electrical installation form an external electrical excitation circuit. The elements of the electrical mounting of the inner electrode are brought out through a special infinitesimal technological hole. The inner electrode is potential, the outer electrode is grounded. Let's believe that the electrodes, shells and elements of electrical installation in their technological properties in no way affect the operation of the transducer - the shell.

After turning on the generator and applying to the electrodes the potential difference $U=U_{0} e^{-i \omega t}$ under the influence of the generated electric field, the stress-strain state of the shell changes. The resulting electric field arising in the volume of the shell consists of both external (which is perturbed by the generator) and internal field, which is caused by displacements from the equilibrium of the ions of the piezoelectric material of the shell (direct piezoelectric effect).

The connection of the main (electric, mechanical and acoustic) physical fields involved in the process of converting electric energy into acoustic, and the circumstances of the problem in terms of technological features and type of electrode necessitate the joint solution of the following equations and the use of the following relations and conditions [2, 6, 14]:

- state equations for piezoceramics;

- equations of motion of material points of a spherical thin shell (or Newton's second law): 


$$
\frac{1}{R_{1}}\left(\sigma_{\varphi \varphi}+\sigma_{\theta \theta}\right)+\rho_{m} \omega^{2} u_{r}=0
$$

where, $\sigma_{\varphi \varphi}, \sigma_{\theta \theta}$ - amplitude values of mechanical compressive-tensile tensions along the axes of azimuthal (latitudinal) and polar (meridional) angles of a spherical coordinate system; $\rho_{m}$ - density of piezoceramics; $u_{r}$ - amplitude value of a harmonically variable radial component of the centrally symmetric vector of displacement of material particles of piezoceramics;

- generalized Hooke's law, written for environments with complex properties:

$$
\begin{aligned}
& \sigma_{r r}=c_{11}^{E} \varepsilon_{r r}+c_{12}^{E} \varepsilon_{\varphi \varphi}+c_{12}^{E} \varepsilon_{\theta \theta}-e_{11} E_{r}, \\
& \sigma_{\varphi \varphi}=c_{12}^{E} \varepsilon_{r r}+c_{22}^{E} \varepsilon_{\varphi \varphi}+c_{12}^{E} \varepsilon_{\theta \theta}-e_{12} E_{r}, \\
& \sigma_{\theta \theta}=c_{12}^{E} \varepsilon_{r r}+c_{12}^{E} \varepsilon_{\varphi \varphi}+c_{22}^{E} \varepsilon_{\theta \theta}-e_{12} E_{r},
\end{aligned}
$$

where $\varepsilon_{r r}, \varepsilon_{\varphi \varphi}, \varepsilon_{\theta \theta}$ - amplitudes of compression-tension deformations along the corresponding axes of the spherical coordinate system; $c_{11}^{E}, c_{12}^{E}$ - components of the matrix of the modulus of elasticity; $e_{11}, e_{12}$ - components of a matrix of piezomodules;

- Cauchy relations for deformations of the form:

$$
\varepsilon_{r r}=u_{r} / r
$$

and

$$
\varepsilon_{\varphi \varphi}=\varepsilon_{\theta \theta}=u_{r} / r
$$

- Helmholtz equation for an acoustic field written in spherical coordinates:

$$
\Delta p_{a}(r, \varphi, \theta)+k^{2} p_{a}(r, \varphi, \theta)=0,
$$

where $k=\omega / c_{0}$ - wave number; $\omega=2 \pi f$ - stake frequency $(\mathrm{rad}) ; f$ - frequency $(\mathrm{Hz})$.

Due to the selected type of electrode, the radiation is undirected. Therefore, the solution of equation (6) will depend only on the radial coordinate $r$ :

$$
p(r, \varphi, \theta, i)=p_{a}(r, i)=\frac{p_{a}}{r} e^{-i(\omega i-k r)}
$$

and the radial component of the displacement vector is related to the pressure and the normal component of the vector of the oscillatory velocity of the points on the surface of the sphere $v_{r}(r, t)$ by the following condition:

$$
\left.v_{r}(r, i)\right|_{r=R_{1}}=-\left.\frac{1}{i \omega \rho_{0}} \frac{\partial p_{a}(r)}{\partial r}\right|_{r=R_{1}}=-\left.i \omega u_{r}\right|_{r=R_{1}} .
$$

Thus the compatible solution of equations (1)-(6) should occur with involvement of acoustic mechanical boundary conditions which are formulated and applied as conditions of conjugation of force type:

$$
\left.\sigma_{i i}\right|_{r=R_{1}}=p=\Delta p=\left.p_{\text {out }}\right|_{r=R_{1}}-\left.p_{\text {in }}\right|_{r=R}
$$

when

$$
\left(r=R+h_{s}\right)
$$

The list ends with the condition of orthogonality of trigonometric and spherical wave functions and the Sommerfeld condition. 
Regarding the boundary conditions for the electric field, let's note that in accordance with $[2,6,14]$ they determine the following situations:

- the first concerns the imposition of the condition of the absence of free electrons in the shell material $\operatorname{div} D=0$ ( $D$ - vector of electrical induction);

- the second - the conditions of connection of the electric field strength in the volume of the transducer with the electric voltage $E=-\operatorname{grad} \Psi,(\Psi-$ electric potential, $\Delta \Psi$ - potential difference);

- the third - the condition illustrating communication of an electric current $I$ and surface density of charges on the electroded surfaces of a cover $Q: I=-i \omega Q$.

Note that all the above characteristics of the main physical fields - electric, mechanical and acoustic are functions of frequency and distance. This dependence (to simplify the presentation) is not shown in the intermediate results and is given only on the final relations, where the search for values gives the amplitude-frequency dependence of some factors. The distance can be fixed.

Thus, using the results of the statement, let's note that the frequency dependencies of the transmittance of the transducer (electric voltage - acoustic pressure) $K_{t}(\omega)$, as well as the frequency dependencies of pressure $p_{a}(\omega)$ and electrical impedance $Z_{e l}(\omega)$ are to be determined.

\section{2. The solving of radiation problem}

Thus, the external load of the spherical shell is a liquid. In the middle of the shell is a vacuum. Consequently, the sound field in the middle of the sphere cannot exist and is not taken into account. The load on the sphere is reduced to the pressure difference $\Delta p_{a}$ on its outer and inner surfaces $\Delta p=\left.p_{\text {out }}\right|_{r=R 1}-\left.p_{\text {in }}\right|_{r=R}$, one of which is zero $\left.p_{i n}\right|_{r=R}$, due to the vacuum condition.

Let's apply condition (8). For the amplitude, of the normal component of mechanical tensions, the force conjugation force is valid:

$$
\left.\sigma_{r r}\right|_{r=R_{1}}=\left.p\right|_{r=R_{1}}=\left.\frac{p_{a}}{r} e^{-i(\omega t-i k r)}\right|_{r=R_{1}},
$$

where $p_{a}$ - pressure amplitude; and the kinetic condition of the conjugation:

$$
\left.v_{r}\right|_{r=R_{1}}=\left.\frac{1}{i \omega \rho} \frac{\partial p_{a}}{\partial r}\right|_{r=R_{1}}=\left.v_{n}\right|_{r=R_{1}}
$$

$v_{r}, v_{n}$ - the velocity of displacement in the radial direction of the material points of the shell surface and the velocity of the normal particles of the medium at the boundary $r=R_{1}$ respectively.

2. 3. Determination of the frequency response of the transmission factor $K_{t}(\omega)$ and electrical impedance $Z_{e l}(\omega)$

Applying condition (10), after substitution in equation (2) let's find the amplitude value of the deformation $\varepsilon_{r r}$ :

$$
\varepsilon_{r r}=\frac{p_{a}}{c_{11}^{E}}-\frac{c_{12}^{E}}{c_{11}^{E}}\left(\varepsilon_{\varphi \varphi}+\varepsilon_{\theta \theta}\right)-\frac{e_{11}}{c_{11}^{E}} E_{r}
$$

After a series of transformations associated with the exclusion of deformations $\varepsilon_{r r}$ from equations (3), (4), for the normal components of mechanical tensions $\varepsilon_{\varphi \varphi}$ and $\varepsilon_{\theta \theta}$ let's obtain:

$$
\begin{aligned}
& \varepsilon_{\varphi \varphi}=c_{11} p_{a}+c_{22} \varepsilon_{\varphi \varphi}+c_{12} \varepsilon_{\theta \theta}-e_{11}^{*} E_{r}, \\
& \varepsilon_{\theta \theta}=c_{11} p_{a}+c_{12} \varepsilon_{\varphi \varphi}+c_{22} \varepsilon_{\theta \theta}-e_{12}^{*} E_{r},
\end{aligned}
$$

where $c_{11}=\frac{c_{12}^{E}}{c_{11}^{E}}, c_{12}=c_{11}^{E}\left(1-\frac{c_{12}^{E}}{c_{11}^{E}}\right), c_{22}=\left(c_{22}^{E}-\frac{\left(c_{12}^{E}\right)^{2}}{c_{11}^{E}}\right), e_{12}^{*}=\left(-e_{11} \frac{c_{12}^{E}}{c_{11}^{E}}+e_{12}^{*}\right)-$ material (15) constants. 
Further, taking into account the relationship between displacements and deformations, which is determined by the Cauchy ratios [2]:

$$
\varepsilon_{\varphi \varphi}=\varepsilon_{\theta \theta}=u_{r} / R
$$

Expressions (13), (14) can be represented as:

$$
\varepsilon_{\varphi \varphi}=\varepsilon_{\theta \theta}=c_{11} p_{a}+Y \frac{u_{r}}{R}-e_{12}^{*} E_{r}
$$

where $Y=\left(c_{12}+c_{22}\right)$ - effective modulus of elasticity.

The symmetrical deformation of the spherical shell, which occurs when it is excited by an external generator, leads to the fact that the electric induction vector $D$ is completely determined by its radial component $D_{r}$ and in accordance with [6] for the sphere is written as:

$$
D_{r}=e_{11} \varepsilon_{r r}+e_{12}\left(\varepsilon_{\varphi \varphi}+\varepsilon_{\theta \theta}\right)+\chi_{11}^{\sigma} E_{r}
$$

where $\chi_{11}^{\sigma}=\chi_{11}^{\varepsilon}+e_{11}^{2} / c_{11}^{E}-$ components of the dielectric constant, set at zero (constant) normal mechanical tensions $\sigma_{r r}$ and deformations $\varepsilon_{r r}$.

After substituting expression (10) for deformations $\varepsilon_{r r}$ in expression (16), and using expression (17), let's change the record for the vector component $D_{r}$ :

$$
D_{r}=2 \frac{u_{r}}{R} e_{12}^{*}+e_{1} p_{a}+\chi_{11}^{\sigma} E_{r}
$$

where

$$
e_{12}^{*}=\left(e_{12}-e_{11} \frac{c_{12}^{E}}{c_{11}^{E}}\right), \chi_{11}^{\sigma}=\left(\chi_{11}^{\varepsilon}+\frac{\left(e_{11}\right)^{2}}{c_{11}^{E}}\right), e_{1}=\frac{e_{11}}{c_{11}^{E}}
$$

Let's apply boundary conditions on an electric field concerning induction $\operatorname{div} D=0[2,6,9,14]$ which defines absence of free carriers of electricity in piezomaterial and in spherical coordinates makes equality:

$$
\operatorname{div} \vec{D}_{r}=\frac{1}{r^{2}} \frac{\partial}{\partial r}\left(r^{2} \vec{D}_{r}\right)=0
$$

For the electric field strength in the volume of ceramics and the potential difference at the electrodes of the shell, let's use the second condition [2]:

$$
U=\int_{R}^{R_{1}} E_{r} \mathrm{~d} r=\Delta \Psi,\left.\psi(r)\right|_{r=R}=U_{0},\left.\psi(r)\right|_{r=R_{1}}=0 .
$$

Regarding the determination of current, let's write the relationship of electrical characteristics - as follows:

$$
Q=\int_{S} D_{r} \mathrm{~d} S, I=i \omega Q
$$

where $Q$ surface density of electric charges on the electrode with area $S$.

The solution of the differential equation (21) in the standard form will be:

$$
\vec{D}_{r}=\left.\frac{C_{02}}{R_{1}^{2}}\right|_{r=R_{1}}
$$

where $C_{02}-$ unknown constant to be found. 
Expression (19), substitute in (24), then let's obtain the equality:

$$
D_{r}=2 \frac{u_{r}}{R} e_{12}^{*}+e_{1} p_{a}+\chi_{11}^{\sigma} E_{r}=\frac{C_{02}}{R^{2}}
$$

whence let's find tension $E_{r}$ :

$$
E_{r}=\frac{1}{\chi_{11}^{\sigma}}\left(\frac{C_{02}}{R^{2}}-2 e_{12}^{*} \frac{u_{r}}{R}-e_{1} p_{a}\right)
$$

Substitute (26) in equations (16), (17) and for the mechanical field let's obtain:

$$
\sigma_{\varphi \varphi}=\sigma_{\theta \theta}=Y^{D} \frac{u_{r}}{R}+Y_{1}^{D} p_{a}-\frac{e_{12}^{*} C_{02}}{\chi_{11}^{\sigma} R^{2}},
$$

where $Y^{D}=Y+2 \frac{e_{12}^{*}}{\chi_{11}^{\sigma}}, Y_{1}^{D}=c_{11}+e_{1} \frac{e_{12}^{*}}{\chi_{11}^{\sigma}}-$ effective modulus of elasticity, which is determined taking into account the interconnectedness of electric and elastic (mechano-acoustic) fields.

Now, in turn, let's use expression (1), which corresponds to Newton's law. Substitution of expression (27) and carrying out a number of transformations gives for the radial component of the movement of material particles of the shell $u_{r}$ :

$$
u_{r}=u_{r}(\omega)=\frac{\frac{2 e_{12}^{*}}{Y^{D} \chi_{11}^{\sigma}} \frac{C_{02}}{R}-2 \frac{Y_{1}^{D}}{Y^{D}} p_{a} R}{\left[2-(\gamma R)^{2}\right]},
$$

where $\gamma^{2}=\rho_{m} \omega^{2} / Y^{D}$ - the square of the wave number of centrally symmetric oscillations of a closed spherical electroelastic shell.

Let's use a group of boundary conditions for the electric field (22):

$$
E_{r}=-\frac{\partial \Psi(r)}{\partial r}=-\frac{\Delta \Psi(r)}{\Delta r}=-\frac{\Psi_{\text {out }}-\Psi_{\text {in }}}{h_{s}}=-\frac{\Psi_{R_{1}}-\Psi_{R}}{h_{s}} .
$$

That is, in the left part of equation (26) let's put condition (29) and let's obtain:

$$
E_{r}=-\frac{\partial \Psi(r)}{\partial r}=\frac{1}{\chi_{11}^{\sigma}}\left(\frac{C_{02}}{R^{2}}-2 e_{12}^{*} \frac{u_{r}}{R}-e_{1} p_{a}\right) \Rightarrow \frac{\partial \Psi(r)}{\partial r}=-\frac{1}{\chi_{11}^{\sigma}} \frac{C_{02}}{R^{2}}+2 e_{12}^{*} \frac{u_{r}}{\chi_{11}^{\sigma} R}+\frac{e_{1}}{\chi_{11}^{\sigma}} p_{a}
$$

Next, in equation (30) let's substitute the expression for the radial component of the displacement $u_{r}$ (expression (28)) and after the introduction of the coefficient of electro-mechanical coupling $k_{e m c}=e_{11}^{*} \sqrt{2 / Y^{D}} \chi_{11}^{\sigma}$ and a number of algebraic transformations:

$$
\frac{\partial \Psi(r)}{\partial r}=-\frac{1}{\chi_{11}^{\sigma}} \frac{C_{02}}{R^{2}}\left\{\frac{\left\{\left(1+k_{e m c}^{2}\right)\left[2-(\gamma R)^{2}\right]-2 k_{e m c}^{2}\right\}}{\left(1+k_{e m c}^{2}\right)\left[2-(\gamma R)^{2}\right]}\right\}+\frac{p_{a}}{\chi_{11}^{\sigma}}\left\{\frac{\left\{\left(1+k_{e m c}^{2}\right)\left[2-(\gamma R)^{2}\right] e_{1}-e_{2} 2 k_{e m c}^{2}\right\}}{\left(1+k_{e m c}^{2}\right)\left[2-(\gamma R)^{2}\right]}\right\} .
$$

Using an integrated representation:

$$
U=\int_{R}^{R+h_{s}} E_{r} \mathrm{~d} r,
$$

integrate the left and right parts (31) on $r$ for the situation $r=R, r=R_{1}$ and get for the electric potential: 


$$
\begin{aligned}
& \left.\Psi(r)\right|_{r=R_{1}}=-\frac{r}{\chi_{11}^{\sigma}} \frac{C_{02}}{R^{2}}\left\{\frac{\left\{\begin{array}{l}
\left(1+k_{e m c}^{2}\right) \times \\
\times\left[2-(\gamma R)^{2}\right]-2 k_{e m c}^{2}
\end{array}\right\}}{\left(1+k_{e m c}^{2}\right)\left[2-(\gamma R)^{2}\right]}\right\}+\left.\frac{p_{a} r}{\chi_{11}^{\sigma}}\left\{\frac{\left\{\begin{array}{l}
\left(1+k_{e m c}^{2}\right) \times \\
\times\left[2-(\gamma R)^{2}\right] e_{1}-e_{2} 2 k_{e m c}^{2}
\end{array}\right\}}{\left(1+k_{e m c}^{2}\right)\left[2-(\gamma R)^{2}\right]}\right]\right|_{R} ^{R_{1}}+C_{01}= \\
& =-\frac{h_{s}}{\chi_{11}^{\sigma}} \frac{C_{02}}{R^{2}}\left\{\frac{\left\{\left(1+k_{e m c}^{2}\right)\left[2-(\gamma R)^{2}\right]-2 k_{e m c}^{2}\right\}}{\left(1+k_{e m c}^{2}\right)\left[2-(\gamma R)^{2}\right]}\right\}+\frac{p_{a} h_{s}}{\chi_{11}^{\sigma}}\left\{\frac{\left\{\left(1+k_{e m c}^{2}\right)\left[2-(\gamma R)^{2}\right] e_{1}-e_{2} 2 k_{e m c}^{2}\right\}}{\left(1+k_{e m c}^{2}\right)\left[2-(\gamma R)^{2}\right]}\right\},
\end{aligned}
$$

where $C_{01}=0$.

The obtained results of determining the electric voltage correspond to the boundary conditions of the form (22) selected during the statement of the problem.

The unknown coefficient $C_{02}$ after transformations can be written as:

$$
C_{02}=U_{0} \frac{\chi_{11}^{\sigma}}{h_{s}} R^{2}\left\{\frac{\left(1+k_{e m c}^{2}\right)\left[2-(\gamma R)^{2}\right]}{\left\{\left(1+k_{e m c}^{2}\right)\left[2-(\gamma R)^{2}\right]-2 k_{e m c}^{2}\right\}}\right\}-p_{a} R^{2}\left\{\frac{\left\{\left(1+k_{\text {emc }}^{2}\right)\left[2-(\gamma R)^{2}\right] e_{1}-e_{2} 2 k_{e m c}^{2}\right\}}{\left\{\left(1+k_{\text {emc }}^{2}\right)\left[2-(\gamma R)^{2}\right]-2 k_{\text {emc }}^{2}\right\}}\right\} .
$$

Therefore, applying the conditions (28) and the found coefficient C02 (34), it is possible to calculate the amplitudes of the displacements of the material points of the surface of the spherical shell. In addition, the use of (34), (23) and Ohm's law for the circuit section can find the value of the electrical impedance of the transducer-shell $Z_{e l}$ :

$$
\begin{gathered}
Z_{e l}=-\frac{U_{0}}{I}=-\frac{U_{0}}{i \omega 4 \pi R^{2}} \frac{1}{D_{z}}= \\
=\frac{U_{0}}{i \omega 4 \pi} \frac{\left\{\left(1+k_{e m c}^{2}\right)\left[2-(\gamma R)^{2}\right]-2 k_{e m c}^{2}\right\}}{\frac{U_{0} \chi_{11}^{\sigma}}{h_{s}} R^{2}\left(1+k_{e m c}^{2}\right)\left[2-(\gamma R)^{2}\right]-p_{a} R^{2}\left\{\left(1+k_{\text {emc }}^{2}\right)\left[2-(\gamma R)^{2}\right] e_{1}-2 e_{2} k_{e m c}^{2}\right\}},
\end{gathered}
$$

or after the introduction of electrical static capacity of the spherical shell:

$$
\begin{gathered}
C_{0}=\frac{i \omega 4 \pi R^{2} \chi_{11}^{\sigma}}{h_{s}}, \\
Z_{e l}=-\frac{U_{0}}{i \omega C_{0}} \frac{\left\{\left(1+k_{e m c}^{2}\right)\left[2-(\gamma R)^{2}\right]-2 k_{e m c}^{2}\right\}}{U_{0}\left(1+k_{e m c}^{2}\right)\left[2-(\gamma R)^{2}\right]-i \omega 4 \pi p_{a} R^{2}\left\{\left(1+k_{e m c}^{2}\right)\left[2-(\gamma R)^{2}\right] e_{1}-2 e_{2} k_{e m c}^{2}\right\}} .
\end{gathered}
$$

In the case where the external environment is a vacuum, the situation is greatly simplified. In this case $\sigma_{r r}=0, p_{a}=0$ and the expression for the impedance (36) takes the form:

$$
Z_{e l}=-\frac{1}{i \omega C_{0}} \frac{\left\{\left(1+k_{e m c}^{2}\right)\left[2-(\gamma R)^{2}\right]-2 k_{e m c}^{2}\right\}}{\left(1+k_{e m c}^{2}\right)\left[2-(\gamma R)^{2}\right]}=-\frac{1}{i \omega C_{0}} L_{0}(\omega),
$$

where

$$
L_{0}(\omega)=\frac{\left\{\left(1+k_{e m c}^{2}\right)\left[2-(\gamma R)^{2}\right]-2 k_{e m c}^{2}\right\}}{\left(1+k_{e m c}^{2}\right)\left[2-(\gamma R)^{2}\right]},
$$

and has the content of the frequency-dependent transfer coefficient of the transducer $K_{t}(\omega)$ in a circle «generator - working medium» (vacuum). 


\section{4. Determination of acoustic pressure frequency response $p_{a}(\omega)$}

Thus, the above obtained a number of useful relationships. These include equation (28) for the radial component of the displacements of the material particles of the shell $u_{r}$, expression for the coefficient $C_{02}$ (34), kinematic conjugation condition (11), properties of orthogonality of spherical and trigonometric wave functions and solution of the Helmholtz equation (6) for the case of realization of zero mode of shell oscillations of known type:

$$
p_{a}(r)=\frac{p_{0}}{r} e^{i k r}
$$

Let's apply the relationship of oscillating speed with displacement and acoustic pressure of the species:

$$
v(r)=-i \omega u_{r}=v_{r}(r)=\frac{1}{i \omega \rho} \frac{\partial p_{0}(r)}{\partial r}
$$

and after substituting in (28) expressions (34), (39), fulfilling the kinematic conditions of conjugation (11) and equality (40), after the transformations let's obtain the amplitude of the acoustic pressure $p_{a}(r)$ at $r=R_{1}$ recorded for the frequency range $\omega$ as:

$$
p_{0}(\omega)=\frac{\omega^{2} \rho U_{0} L_{1}(\omega) R_{1}^{2}}{\left(1+\omega^{2} \rho\left(L_{2}(\omega)+L_{3}(\omega)\right)\right)},
$$

where

$$
\begin{gathered}
L_{1}(\omega)=\left(\frac{\chi_{11}^{\sigma} R_{1}^{2}}{h_{s}}\right)\left(\frac{2 e_{12}^{*}}{\chi_{11}^{\sigma} Y^{D} R_{1}}\right)\left(\frac{F_{1}(\omega)}{F_{3}(\omega)}\right), \\
L_{2}(\omega)=\left(R_{1}^{2}\right)\left(\frac{2 e_{12}^{*}}{\chi_{11}^{\sigma} Y^{D} R_{1}}\right)\left(\frac{F_{2}(\omega)}{F_{3}(\omega)}\right), \\
L_{3}(\omega)=\left(R_{1}^{2}\right)\left(\frac{2 Y_{1}^{D} R_{1}}{Y^{D}}\right)\left(\frac{1}{F_{3}(\omega)}\right), \\
F_{1}(\omega)=\frac{\left\{\left(1+k_{\text {emc }}^{2}\right)\left[2-\left(\gamma R_{1}\right)^{2}\right]-2 k_{e m c}^{2}\right\}}{\left(1+k_{e m c}^{2}\right)\left[2-\left(\gamma R_{1}\right)^{2}\right]}, \\
F_{2}(\omega)=\frac{\left\{\left(1+k_{e m c}^{2}\right)\left[2-\left(\gamma R_{1}\right)^{2}\right] e_{1}-2 e_{2} k_{e m c}^{2}\right\}}{\left(1+k_{\text {emc }}^{2}\right)\left[2-\left(\gamma R_{1}\right)^{2}\right]}, \\
F_{3}(\omega)=\left[2-\left(\gamma R_{1}\right)^{2}\right] .
\end{gathered}
$$

The actual acoustic pressure $p_{a}(\omega)$ is determined by using the expression for the pressure amplitude (41), (42) in equation $p_{0}(\omega)(39)$.

\section{5. Experiment}

The obtained theoretical results are confirmed experimentally in terms of measuring the acoustic pressure created by the transducer in the free field $p_{a}(\omega)$.

The measurement results $p_{a}(\omega)$ are represented by frequency characteristics in the frequency range of the calculated part. Evolutions were carried out in the measuring basin of the State Enterprise Research Institute «Hydroprylad» in Kyiv, according to the scheme (Fig. 2).

The working space during the measurements corresponded to the conditions of the far field, and in the receiving path the method of gating the useful signal by time and distance was implemented. At the same time the relation was carried out: $U_{S} / U_{D} \geq 15 \mathrm{~dB}$, where $U_{S}-$ electric voltage on the recorder of the useful signal, $U_{D}$ - electric voltage of damage. 


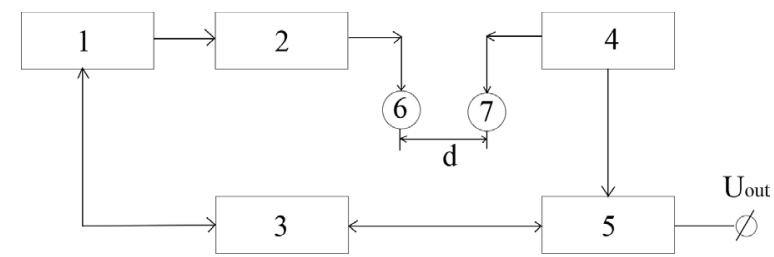

Fig. 2. Measurement scheme: 1 - master generator; 2 - power amplifier; 3 - selector; 4 - amplifier; 5 - bandpass filter; 6 - transformer; 7 - control hydrophone

The results of acoustic pressure measurements at the frequencies of the working range of numerical studies show (Fig. 3) that the frequency dependences of pressures measured in the resonance region of the zero mode of oscillations of the sphere, provided doubling the distance from the emitter to the control hydrophone, decreases by $6 \mathrm{~dB}$. That is, the law of inverse radii is fulfilled, which corresponds to the nature of the calculated curves (Fig. 6).

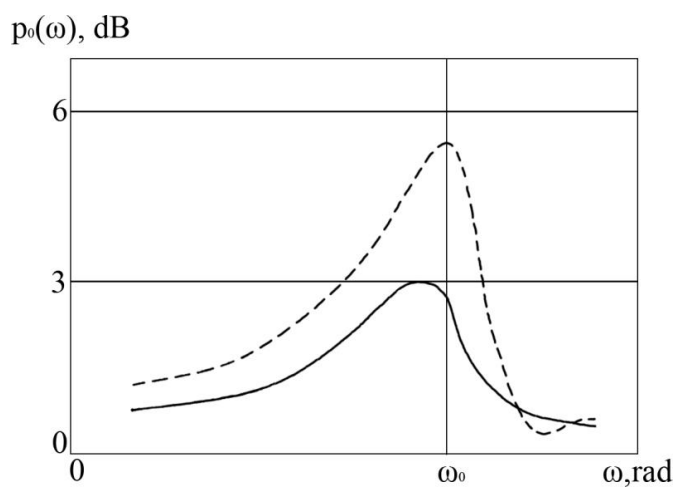

Fig. 3. Amplitude-frequency characteristic of pressure $p(\omega), \mathrm{dB}\left(---d \geq \frac{2 D^{2}}{\lambda},---2 d\right)$

Based on the obtained graphs, let's believe that the theoretical studies correspond to the practical results of the work.

\section{Results and discussion}

Calculations of frequency dependences of coefficients $K_{t}(\omega), Z_{e l}(\omega), p_{a}(\omega)$ were carried out for a spherical cover with radius $R_{1}=0.5 \mathrm{~m}$ which is made of CTS-19 piezoelectric material having coefficient $k_{\text {emc }}=0.5$. Estimated distances for acoustic pressures were $1 \mathrm{~m}$ and $10 \mathrm{~m}$.

The results of calculations are shown in Fig. 4-6.

In Fig. 4, $\boldsymbol{a}$ the calculated graph of $K_{t}(\omega)$ is shown, which has two local extremes - local minimum and local maximum. The local minimum of $K_{t}(\omega)$ determines the situation of the largest amplitudes of displacements of the radial components of the shell movements and is a resonance of the electromechanical nature of the corresponding frequency $\omega_{r}$, for which equality must be satisfied:

$$
2-\left(\gamma R_{1}\right)^{2}-2 k_{e m c}^{2}=2-\left(\frac{\omega_{r}}{\omega_{a}}\right)^{2}=0,
$$

where $\gamma^{2}=\rho_{0} \omega_{r}^{2} / Y^{D}, k_{e m c}^{2}=\left[2-\left(\frac{\omega_{r}}{\omega_{a}}\right)^{2}\right] \frac{1}{2}$ accordingly, the square of the wave number of oscillations of the shell and the square of the coefficient of electromechanical coupling.

The second local extremum determines the situation of the smallest radial displacements of the points of the shell surface and is an electromechanical antiresonance with frequency $\omega_{a}$. The equality $F_{3}(\omega)=\left[2-\left(\gamma R_{1}\right)^{2}\right]$ holds for it. 

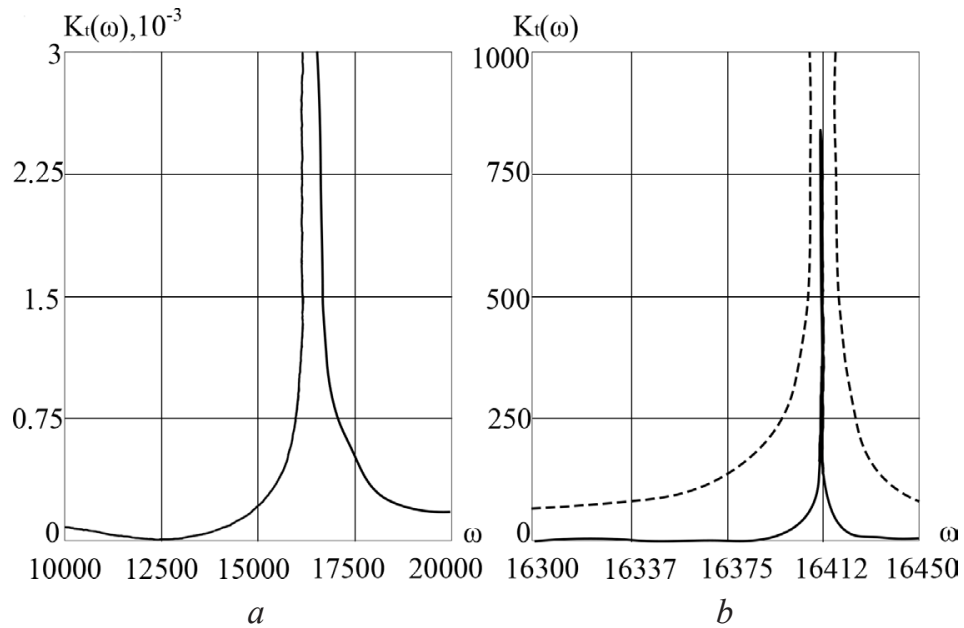

Fig. 4. Frequency response of transmission coefficients $K_{t}(\omega)$ : $a$ - working medium - vacuum;

$$
b \text { - working medium - water }
$$

The modulus of elasticity is calculated by the formulas:

$$
Y^{D}=\frac{\omega_{a}^{2} \rho_{0}}{2} R_{1}, Y=Y^{D} \frac{1}{\left(1+k_{e m c}^{2}\right)} .
$$

In our computational situation, the frequency of electromechanical resonance is $2600 \mathrm{~Hz}$ and the introduction of the load from the working medium (Fig. 4, b) affects the frequency response for the selected computational situations only in part of the active load.

The given situation with change of amplitudes of radial components taking into account connectivity should correspond to change of electric impedance $Z_{e l}(\omega)$ of hydroelectric-elastic system. Indeed (Fig. 5), at the resonance frequency the impedance $Z_{e l}(\omega)$ is small and in the circuit «generator-working medium», there will be a maximum of the current mode, which includes the bias current and the current in the external circuit. The opposite situation is observed at the antiresonance frequency.

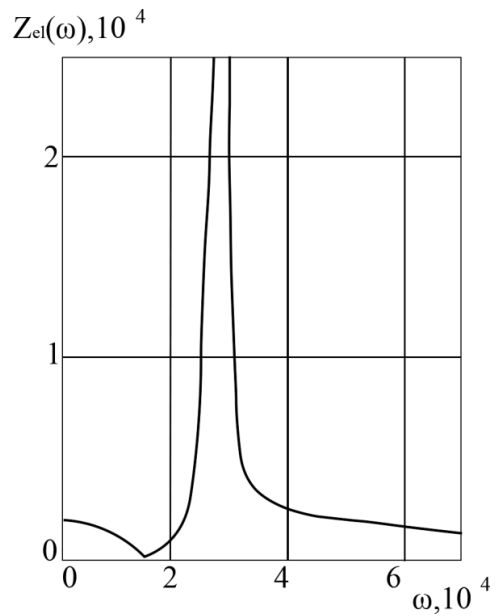

Fig. 5. Frequency response of electrical impedance $Z_{e l}(\omega)$

Regarding the frequency response of the acoustic pressure, it is obvious that the frequency dependence of the pressure is expected. The graphs themselves contain a local maximum, which is at the resonant frequency $\omega_{r}$ of the zero mode of oscillations.

The calculations were performed for two situations: the first - standard, for which the pressure is determined at a traditional distance of $1 \mathrm{~m}$, and the second - at a distance of $10 \mathrm{~m}$. 
In this case, (Fig. 6) the levels of local maxima decrease by $20 \mathrm{~dB}$ with an increase in the calculated distance by 10 times, which is fully consistent with the law of inverse radii and confirmed experimentally (Fig. 3).

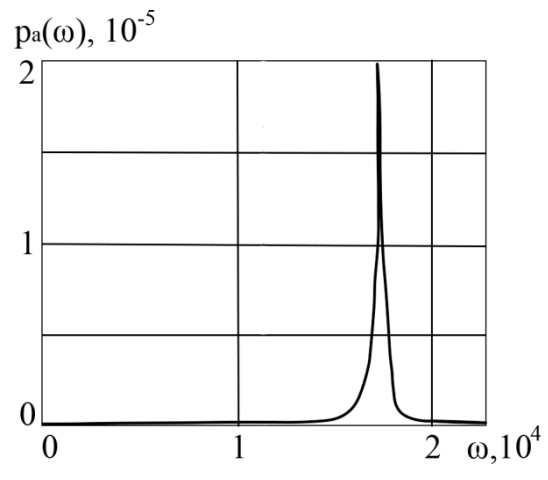

$a$

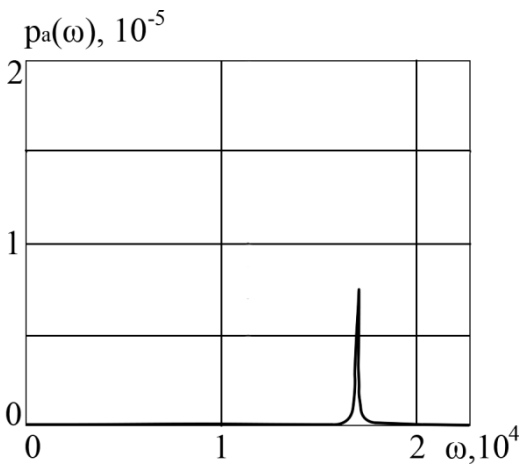

$b$

Fig. 6. Frequency response of acoustic pressure $p_{a}(\omega): a-R_{1}=1 \mathrm{~m} ; b-R_{1}=10 \mathrm{~m}$

The obtained results of using the «pass-through» method in the problem of sound radiation show a new generalizing approach that allows one to obtain its exact solutions in the representation of a transducer - a thin-walled piezoceramic shell. Thus, the result of the formation of a field of zero-order acoustic waves can be described by jointly solving the equations of oscillations of the source, the equation for the acoustic field, the equation of state for piezoelectric ceramics, and the Cauchy relations for displacements and deformations. The key point of the work is the solution within the framework of the theory of thin shells of revolution and the conditions of conjugation in the acoustic, mechanical, and also in the electric field. Let's also note the fact that the traditional method of equivalent circuits [21], which is successfully used in the resonance region of the frequency response of a sound source, worsens the reliability of the numerical results, the more the further from the resonance the frequency region under consideration is.

Features of the proposed solution consist in taking into account, for the considered oscillatory system, the connection of acoustic mechanical elastic forces with forces of electrical nature. In this case, the electric boundary conditions determine the absence of free electric charges in the piezoceramics and the presence of only a dynamic component of current values - in the form of the surface density of electric charges in the region of the electroded surfaces of the shell.

Of course, the proposed formulation and solution have a number of limitations. These include the following:

- restrictions associated with the thickness of the shell wall (it should be no more than $10 \%$ of the radius);

- restrictions associated with the method and type of electroplating of the surfaces of the sphere (full or partial);

- technological limitations associated with the uniformity of electrode deposition and the provision of reliable electrical contact on the inner and outer electrodes (in the work these technological features are idealized and are not taken into account);

- taking into account the possible presence of the throat of the sphere and the associated possible changes in the frequency characteristics of the acoustic field;

- idealization of the setting device cable line.

Some of these restrictions can be removed. First of all, this concerns the features of a constructive and technological nature in terms of electroplating the surfaces of the converter, which play an important role in the formation of its spatial properties and in the future can be considered taking into account the presented material and the worked out tasks [11, 13-16].

Further development of the proposed scientific instrument of the «pass-through» method implies its use in various areas of research into the spatial and energy characteristics of acoustic antennas and transducers as applied not only to the problems of radiation, but also reception. 
This requires the complication of the mathematical apparatus in terms of the special functions used (from cylindrical functions to spheroidal or ellipsoidal - for converters of basic canonical forms and systems based on them), solution methods (from standard methods for solving differential equations to methods for solving infinite systems with complex terms), issues of convergence of complex series and conditions of electrical and acoustic loading of transducers, as well as the presence of acoustic screens, methods of location and connection of transducers, etc.

In the development of the proposed topic, after modification of the formulations of the corresponding equations and boundary conditions, it is assumed that the «pass-through» method will provide a broader physical interpretation of the effects of the interaction of acoustic, mechanical and electric fields of the transducer and their influence on the spatial and energy properties of transducers and systems based on them. This concerns the replacement of the boundary conditions by the conjugation conditions and the description of methods for forming the spatial characteristics of single transducers and their systems for various types of electroding of transducers, as well as the type and nature of electrical and acoustic loads.

\section{Conclusions}

The application of the method of the end-to-end problem of the direction «hydroelectric elasticity» in the situation of sound radiation shown on the example of operation of a spherical electroacoustic source of zero order in an ideal liquid. The electroacoustic emitter source is supplied by a spherical piezoceramic thin-walled shell with complete electroding of its surfaces.

In terms of the load of the transducer from the working environment shows, the possibility, in fact, the solution of the radiation problem taking into account the effects of connectivity of the main physical fields, which are characterized by elastic forces of mechano-acoustic nature and Coulomb forces (electric forces).

The analysis of the main frequency dependences of complex characteristics of the emitter is calculated and made: $K_{t}(\omega), Z_{e l}(\omega), p_{a}(\omega)$. Possibilities of a decision in part of definition of material constants of piezomaterial of a cover of the fixed geometrical sizes and frequency dependences of the basic electroacoustic characteristics of a source are shown.

It is determined that this approach is promising and should be extended to the problem of radiation-reception of sound, within the wave acoustics of infinite and bounded spaces.

\section{Acknowledgments}

The work was performed within the initiative scientific topic: «Problems of underwater sound communication», registration number in KPI named after Igor Sikorsky FEL - 1/3, state registration number № 0113U008183.

\section{References}

[1] Mason, W. P. (1950). Piezoelectric crystals and their application to ultrasonics. Van Nostrand, 508.

[2] Grinchenko, V. T., Ulitko, A. F., Shul'ga, N. A. (1989). Mekhanika svyazannyh poley v elementah konstruktsiy. Vol. 5. Elektrouprugost'. Kuiv: Nauk. dumka, 280.

[3] Ustinov, Yu. A. (1996). Electroelasticity. Fundamentals of the theory and some applications. Sorosovskiy obrazovatel'niy zhurnal, 3, 122-127. Available at: https://web.archive.org/web/20060828163531/http://journal.issep.rssi.ru/articles/ pdf/9603_122.pdf

[4] Aronov, B. (2009). Coupled vibration analysis of the thin-walled cylindrical piezoelectric ceramic transducers. The Journal of the Acoustical Society of America, 125 (2), 803-818. doi: https://doi.org/10.1121/1.3056560

[5] Shul'ga, N. A., Grigorenko, A. Y., Loza, I. A. (1984). Axisymmetric electroelastic waves in a hollow piezoelectric ceramic cylinder. Soviet Applied Mechanics, 20 (1), 23-28. doi: https://doi.org/10.1007/bf00883567

[6] Petrishchev, O. N. (2012). Harmonic oscillations of piezoceramic elements. Part 1. Harmonic oscillations of piezoceramic elements in vacuum and the resonance-antiresonance method. AVERS.

[7] Babaev, A. E., Leyko, A. A., Savin, V. G. (1989). Akusticheskie i mekhanicheskie polya radial'no polyarizovannogo tsilindricheskogo vibratora pri impul'snom elektricheskom vozbuzhdenii. Akusticheskiy zhurnal, 35 (2), 211-217.

[8] Morgun, I. O., Savin, V. G. (2007). Acoustic-to-electric pulse transforming by a spherical pieziceramic shell baffled with an external elastic shell. Akustychniy visnyk, 10 (3), 60-69. Available at: http://dspace.nbuv.gov.ua/handle/123456789/1047 
[9] Derepa, A., Dzhanazian, V., Leiko, O., Drozdenko, O. (2019). Modern approaches to the design of hydroacoustic antennas of ship hydroacoustic stations. Weapons and military equipment, 22 (2), 93-98. doi: https://doi.org/10.34169/2414-0651.2019.2(22).93-98

[10] Korzhyk, O. V. (2010). Application of the «through» problem to the study of the amplitude-frequency characteristics of the acoustic field of a receiving cylindrical piezoceramic transducer with cut-off electrodes. Elektronika ta zv'yazok, 15(3), 160-166.

[11] Korzhyk, A. V., Kuroiedova, T. S., Philippova, N. Y. (2013). The analysis of electromechanical characteristics of radiating cylindrical piezoceramic transducer with surface coated by solid elec-trodes located in the closed ring layer. Electronics and Communications, 18 (1), 102-109. doi: https://doi.org/10.20535/2312-1807.2013.18.1.189189

[12] Novak, D. D., Korzhyk, O. V., Petryshchev, O. M., Hubinets, Yu. V. (2014). The output voltage determination on the load of piezoelastic spherical transducer with acoustic medium inside (the theoretical basis and solution). Information Processing Systems, 7 (123), 49-56.

[13] Leiko, O. H., Sviatnenko, A. O. (2017). Physical fields aimed sonar antennas based on cylindrical emitters with internal screens. Electronics and Communications, 22 (2), 66-72. doi: https://doi.org/10.20535/2312-1807.2017.22.1.86484

[14] Korzhyk, O. V. (2013). To the boundary conditions for problem of sound receiving by spherical electroelastic transduser with disconnecting electrodes. Electronics and Communications, 18 (2), 97-103. doi: https://doi.org/10.20535/2312-1807. 2013.18.2.186872

[15] Aronov, B., Brown, D. A., Yan, X., Bachand, C. L. (2011). Modal analysis of the electromechanical conversion in piezoelectric ceramic spherical shells. The Journal of the Acoustical Society of America, 130 (2), 753-763. doi: https://doi.org/ $10.1121 / 1.3593364$

[16] Leiko, O., Derepa, A., Pozdniakova, O., Starovoit, Y. (2018). Acoustic fields of circular cylindrical hydroacoustic systems with a screen formed from cylindrical piezoceramic radiators. Romanian Journal of Acoustics and Vibration, 15 (1), 41-46. Available at: http://rjav.sra.ro/index.php/rjav/article/view/49

[17] Derepa, A. V., Leiko, O. H., Drozdenko, O. I., Svyatnenko, A. O. (2019). Properties of electric fields of hydroacoustic radiators with internal screens. Ozbroyennya ta viys'kova tekhnika, 4 (24), 41-48. Available at: http://nbuv.gov.ua/UJRN/ovt_2019_4_6

[18] Starovoit, Y. I., Kurdiuk, S. V., Leiko, O. H. (2018). Physical fields of hydroacoustic sonar arrays with baffle and cylindrical piezoceramic radiators with radial polarization. Microsystems, Electronics and Acoustics, 23 (1), 30-36. doi: https://doi.org/10.20535/2523-4455.2018.23.1.99725

[19] Shyshkova, K. A., Leiko, O. H. (2019). Radiation of Sound by a Cylindrical Piezoceramic Converter with Radial Polarization and a Rigid Screen. Microsystems, Electronics and Acoustics, 24 (4), 68-73. doi: https://doi.org/10.20535/ 2523-4455.2019.24.4.184027

[20] Philippova, N. (2014). Setting and solution problem of sound emission of multimode piezoceramic transducer, placed in a closed loop layer. Vestnik Zabaykal'skogo gosudarstvennogo universiteta, 01 (104), 74-82.

[21] Aronov, B. S. (1990). Elektromekhanicheskie preobrazovateli iz p'ezoelektricheskoy keramiki. Leningrad: Energoatomizdat.

Received date 03.08.2020

Accepted date 06.07.2021

Published date 13.09.2021
(C) The Author(s) 2021

This is an open access article under the Creative Commons CC BY license

How to cite: Korzhyk, O., Naida, S., Kurdiuk, S., Nizhynska, V., Korzhyk, M., Naida, A. (2021). Use of the pass-through method to solve sound radiation problems of a spherical electro-elastic source of zero order. EUREKA: Physics and Engineering, 5, $133-146$. doi: https://doi.org/10.21303/2461-4262.2021.001292 